\title{
Acoso sexual: Elementos de discusión para la redefinición del fenómeno
}

Víctor Manuel Aguilar Pereira ${ }^{1}$ y Ligia María Orellana Calderón²

1. Estudiante de Licenciatura en Psicología, Col. Santa Matilde, Calle Castro Morán \#63, San Ramón, Mejicanos, San Salvador, El Salvador. E-mail: victorxaguilar@gmail.com

2. Estudiante de Licenciatura en Psicología, Universidad Centroamericana José Simeón Cañas (UCA), Boulevard Los Próceres, San Salvador, El Salvador. E-mail: Im.orca@gmail.com

Avenida Las Anturias \#123, colonia Miramonte poniente 2, San Salvador, El Salvador.

Recibido: septiembre de 2006 / Aceptado: octubre de 2006

LA PROBLEMÁTICA DEL ACOSO SEXUAL SE HA CONFINADO, GENERALMENTE, al ámbito laboral, y se ha considerado como un efecto de relaciones jerárquicas. Esta concepción ha limitado su estudio en cierta medida, y ha permitido que se ignoren otras manifestaciones, mucho más sutiles, pero que, con una mirada más minuciosa y crítica, dejan entrever los cimientos socio-históricos de este fenómeno: la arraigada ideología de género, traducida en violencia de género, que mancilla diariamente los derechos fundamentales de la mujer. Si bien falta un largo camino por recorrer para reformular una definición integral de acoso sexual, los factores aquí presentados se han considerado elementos primordiales para esta reformulación, puesto que permitirán un conocimiento más amplio de las raíces que sustentan al acoso sexual, lo que a su vez encaminará propuestas de acción efectivas para que, tanto hombres como mujeres, puedan hacerle frente.

Palabras clave: Acoso sexual / mujeres-cuestiones sociales y morales / violencia contra la mujer

Este artículo constituye una revisión de la literatura referente al fenómeno del acoso sexual, y la presentación de aquellos elementos que, a juicio de los autores, se deben tomar en cuenta para una futura redefinición de dicho fenómeno. La información proveniente de disciplinas como la psicología y la sociología es el núcleo de esta investigación bibliográfica y, a partir de ella, se realiza un análisis psicosocial de esta problemática. Este enfoque se ha elegido por dos razones fundamentales: primero, porque esta perspectiva logra superar los reduccionismos sociologistas o psicologistas en que a menudo incurren las explicaciones sobre los distintos aspectos de la vida de los seres humanos; segundo, por su enfoque en los objetivos de desideologización de la realidad y de la reivindicación de los derechos humanos, y su compromiso con la liberación de los grupos oprimidos.

De esta manera, uno de los fines más importantes que persigue este trabajo es llamar la atención, tanto de la comunidad científica como la sociedad en general, hacia una 
problemática común, seria y, aún así, poco tratada. Esto, ya que a pesar del notable aumento del interés por abordar la problemática de la violencia en general, y los crecientes esfuerzos por atender particularmente la violencia de género, el fenómeno del acoso sexual permanece invisibilizado en la realidad en que vivimos, y relegado a un segundo o tercer plano en las investigaciones y publicaciones sobre violencia.

\section{Extensión, gravedad y relevancia del problema}

La extensión del acoso sexual, principalmente en la población femenina, se puede constatar a través de las distintas cifras que han venido reflejando las investigaciones. A nivel global, según los datos que reporta la Organización Panamericana de la Salud (OPS, 2003b), el 33\% de las mujeres entre 16 y 49 años ha sido víctima de acoso sexual. Los Cuadros 1, 2, 3 y 4 muestran resultados de diversos estudios sobre acoso sexual a nivel mundial, desde 1980 hasta 2006. Los tres primeros se refieren a datos obtenidos en Europa, mientras que el cuarto se refiera a América Latina. Las diferencias observadas en las cifras obtenidas por este tipo de estudios son muy grandes; por ejemplo, un estudio realizado en Bélgica, señaló que el porcentaje de mujeres que reportaban haber sufrido distintas modalidades de acoso sexual no sobrepasaba el 2\% (ver Cuadro 3), mientras que, según la OPS (2003b), el 95\% de trabajadoras mexicanas reporta haber sido víctimas de acoso sexual (ver Cuadro 4). 


\section{Cuadro 1: Resultados de algunos estudios sobre acoso sexual en Europa y América del Norte entre 1980 y 1992}

\begin{tabular}{|c|c|c|}
\hline País & Año & Principales Hallazgos \\
\hline $\begin{array}{l}\text { Alemania (ex República } \\
\text { Federal Alemana) }\end{array}$ & 1984 & $\begin{array}{l}-25 \% \text { de las mujeres interrogadas habían sido víctimas de acoso sexual } \\
\text { en el lugar de trabajo }\end{array}$ \\
\hline $\begin{array}{l}\text { Alemania (ex República } \\
\text { Democrática Alemana) }\end{array}$ & 1987 & $\begin{array}{l}\text {-El país reportó a la ONU que en 1987, fueron denunciados } 377 \text { casos } \\
\text { de acoso sexual en los tribunales }\end{array}$ \\
\hline Australia & $1984-1987$ & $\begin{array}{l}\text {-El país reportó a la ONU que } 2,400 \text { casos de discriminación sexual y } \\
\text { acoso sexual fueron tramitados entre } 1984 \text { y } 1987 \text {-Hubieron } 292 \\
\text { demandas por acoso sexual }\end{array}$ \\
\hline Australia & $1990-1991$ & -Se registraron 18 demandas por sexo y acoso sexual \\
\hline Canadá & 1983 & $\begin{array}{l}\text {-Una encuesta realizada por la Comisión Canadiense de los Derechos } \\
\text { de la Persona, reflejo que 1,200,000 mujeres habían sufriso acoso } \\
\text { sexual en algún momento de su vida laboral. }\end{array}$ \\
\hline Canadá Ottawa & 1983 & $\begin{array}{l}\text {-Una encuesta realizada por el Ministerio de Abastecimiento y Servi- } \\
\text { cios, reportó que el } 49 \% \text { de las mujers encuestadas habían experimen- } \\
\text { tado al menos una forma de acoso sexual. }\end{array}$ \\
\hline España & $\mathrm{S} / \mathrm{F}$ & $\begin{array}{l}\text {-La Unión General de Trabajadores, en estudios realizados con sus } \\
\text { afiliadas, encontró que el } 84 \% \text { de las encuestadas manifestaban haber } \\
\text { sufrido acoso sexual alguan vez en el trabajo. }\end{array}$ \\
\hline España & $1986-1989$ & $\begin{array}{l}\text {-El Instituto de la Mujer, a partir de diversas investigaciones realizadas } \\
\text { entre } 1986 \text { y } 1989 \text { reportó que, de las mujeres encuestadas, el } 43 \% \\
\text { experimentó galanteos o insinuaciones no deseadas alguna vez; el } \\
88 \% \text {, hostigamiento sexal sin contacto fiísico; el } 52 \% \text { roces o avances } \\
\text { físicos; el } 25 \% \text { renunció a su trabajo a causa de acoso sexual y como } \\
\text { única forma de evitarlo; y el } 42 \% \text { manifestó que su crecimiento y } \\
\text { promoción se vio postergada por no aceptar proposiciones sexual., }\end{array}$ \\
\hline Estados Unidos & 1980 & $\begin{array}{l}\text {-El Consejo Protector del Servicio Meritorio, en una encuesta realizada } \\
\text { por aproximadamente 18,000 empleadas públicas federales del Servi- } \\
\text { cio Civil, encontró que el } 25 \% \text { había sufrido algun tipo de hostigamien- } \\
\text { to sexual en los dos últimos años. }\end{array}$ \\
\hline Estados Unidos & 1992 & $\begin{array}{l}\text {-Una encuesta nacinaol realizada por el diario "Washington Post" } \\
\text { obtuvo los siguientes resultados } \\
\text { a) El } 7 \% \text { de los hombres y el } 32 \% \text { de las mujeres que realizaron la } \\
\text { encuesta reportaron haber sido víctimas de acoso } \\
\text { b) El } 10 \% \text { de los hombres y el } 36 \% \text { de las mujeres habían padecido } \\
\text { hostigamiento sexual fuera del trabajo } \\
\text { c) El } 49 \% \text { de los hombres y el } 50 \% \text { de las mujeres, sabían de alguien } \\
\text { que había sido víctima de acoso sexual. }\end{array}$ \\
\hline Holanda & 1984 & $\begin{array}{l}\text {-Una encuesta oficial del gobierno encontró que el } 56 \% \text { de las mujeres } \\
\text { había sufrido alguna forma de acoso sexual y que la mayor parte de } \\
\text { ellas lo había sufrido más de una vez. }\end{array}$ \\
\hline Reino Unido & 1982 & $\begin{array}{l}\text {-El } 51 \% \text { de las mujeres manifestaron que habían sido víctimas de } \\
\text { alguna forma de insinuación sexual no deseada en el trabajo durante } \\
\text { el año } 1982 .\end{array}$ \\
\hline Suecia & 1987 & $\begin{array}{l}\text {-La Mediadora para la Igualdad de Oportunidades presentó un informe } \\
\text { de una encuesta realizad entre } 2,000 \text { mujeres, en la que se encontró } \\
\text { que el } 16 \% \text { de las mujeres dijeron haber sido víctimas de acoso Sexual. }\end{array}$ \\
\hline
\end{tabular}


Cuadro 2: Resultados de algunos estudios sobre acoso sexual en Europa entre 1985 y 1994

\begin{tabular}{|c|c|c|c|}
\hline País & $\begin{array}{l}\text { Año de la } \\
\text { investigación }\end{array}$ & $\begin{array}{l}\text { Resultados de } \\
\text { acoso }\end{array}$ & Observaciones sobre la metodología \\
\hline Alemania & 1994 & $72 \%$ mujeres & $\begin{array}{l}\text { Envío de cuestionarios a mujeres sindicalistas, la respuesta } \\
\text { fue de un } 10 \% \text {. Participaron } 1.981 \text { mujeres }\end{array}$ \\
\hline Austria & 1988 & $81 \%$ mujeres & $\begin{array}{l}10.000 \text { cuestionarios que los sindicatos distribuyeron entre } \\
\text { empresas. R espondieron algo menos del } 14 \%\end{array}$ \\
\hline Dinamarca & 1991 & $11 \%$ mujeres & $\begin{array}{l}\text { Muestra estadística } \\
\text { trabajadoras }\end{array}$ \\
\hline Finlandia & 1993 & $\begin{array}{l}27 \% \text { mujeres } \\
30 \% \text { Hombres* }\end{array}$ & $\begin{array}{l}\text { Envío de } 3.000 \text { cuestionarios a personas seleccionadas } \\
\text { aleatoriamente } \\
\text { "E sta cifra tan alta se explica porque la pregunta sobre } \\
\text { acoso indicaba que las situaciones eran "no buscadas" } \\
\text { en vez de "indeseadas" }\end{array}$ \\
\hline Francia & $\begin{array}{l}1985 \\
1988 \\
1990\end{array}$ & $\begin{array}{l}36 \% \text { mujeres } \\
9 \% \text { mujeres } \\
8 \% \text { mujeres }\end{array}$ & $\begin{array}{l}\text { Muestra estadística de } 958 \text { mujeres } \\
\text { Muestra estadística de } 313 \text { mujeres } \\
\text { Muestra estadística de } 804 \text { mujeres } \\
\text { *R eferido a requerimientos sexuales de algún superior }\end{array}$ \\
\hline Holanda & 1994 & $\begin{array}{l}7 \% \text { mujeres* } \\
2 \% \text { hombres* }\end{array}$ & $\begin{array}{l}\text { Cuestionario publicado en } 14 \text { periódicos. } \\
\text { El radio de respuesta osciló entre el } 3 \% \text { y el } \\
13 \% \text {. En total respondieron } 15.000 \text { personas. } \\
\text { *Pregunta directa sobre acoso }\end{array}$ \\
\hline Luxemburgo & 1993 & $\begin{array}{l}78 \% \text { mujeres }^{*} \\
13 \% \text { mujeres }\end{array}$ & $\begin{array}{l}\text { Muestra representativa de } 502 \text { mujeres } \\
\text { * Pregunta a partir de un listado de conductas } \\
* \text { Pregunta directa sobre acoso }\end{array}$ \\
\hline Portugal & 1988 & $\begin{array}{l}34 \% \text { mujeres } \\
7 \% \text { mujeres } \\
7 \% \text { hombres }\end{array}$ & $\begin{array}{l}\text { Muestra estadística de } 1.022 \text { mujeres para la } \\
\text { Administración pública a nivel nacional } \\
708 \text { cuestionarios distribuidos por correo }\end{array}$ \\
\hline Reino Unido & 1993 & $\begin{array}{l}54 \% \text { mujeres } \\
9 \% \text { hombres }\end{array}$ & $\begin{array}{l}1.700 \text { cuestionarios cumplimentados, no se dispone del } \\
\text { porcentaje de respuesta }\end{array}$ \\
\hline Suecia & 1987 & $\begin{array}{l}17 \% \text { mujeres } \\
2 \% \text { mujeres* } \\
1 \% \text { hombres* }\end{array}$ & $\begin{array}{l}\text { Cuestionarios enviados a } 4.000 \text { mujeres sindicalistas, } \\
\text { respondieron el } 50 \% \\
\text { Entre } 10.000 \text { y } 15.000 \text { encuestadas } \\
\text { * Pregunta sobre acoso en los últimos } 3 \text { meses }\end{array}$ \\
\hline
\end{tabular}

Fuente: Comisión Europea "Sexual harassment at the workplace in the European Union” ( Gea 21. S.L. et al., 2000: 24) 
Cuadro 3: Resultados de algunos estudios sobre acoso sexual en Europa entre 1999 y 2003

\begin{tabular}{|c|c|c|}
\hline País & Año & Principales Hallazgos \\
\hline Bélgica & $1999-2003$ & $\begin{array}{l}-1.3 \% \text { de la población es víctima de acoso sexual verbal } \\
-2.0 \% \text { son víctimas de acoso sexual no verbal } \\
-0.6 \text { son víctimas de formas físicas de acoso sexual }\end{array}$ \\
\hline Italia & $1997-1998$ & $\begin{array}{l}-56.3 \% \text { de la población femenina ha sido víctima de acoso sexual verbal } \\
-22.6 \% \text { de la población femenina ha sido víctima de exhibicionismo alguna } \\
\text { vez en la vida (4.2\% de los casos han ocurrido en los últimos } 3 \text { años). } \\
-24 \% \text { de las mujeres ha sido víctima de acoso físico alguna vez en la vida } \\
\text { (5.7\% en los últimos } 3 \text { años). }\end{array}$ \\
\hline Malta & 2002 & $\begin{array}{l}\text {-La incidencia del acoso sexual cometido por un superior fue de } 30.1 \% \text {. } \\
\text {-La prevalencia del acoso sexual verbal por un superior fue de } 7.5 \% \text {. } \\
\text {-La prevalencia del acoso sexual no verbal por un superior fue de } 3.2 \% \text {. }\end{array}$ \\
\hline España & $\mathrm{S} / \mathrm{F}$ & La incidencia varía entre 1-18\% según el nivel de severidad del acoso \\
\hline
\end{tabular}

Fuente: elaboración propia a partir de IPEU-FGS (2004)

Cuadro 4: Resultados de algunos estudios sobre acoso sexual en América Latina entre 1990 y 2005

\begin{tabular}{|c|c|c|}
\hline País & Año & Principales Hallazgos \\
\hline Argentina $^{1}$ & 1994 & $\begin{array}{l}\text { Una investigación realizada por la Unión de Personal Civil de la Nación } \\
\text { (UPCN) con } 302 \text { mujeres trabajadoras, reflejó que } 143(47.4 \%) \text { habían } \\
\text { sufrido acoso sexual de diferentes tipos. }\end{array}$ \\
\hline Chile $^{2}$ & 1991 & $\begin{array}{l}\text { Según los resultados de una encuesta realizada a } 1200 \text { trabajadoras del } \\
\text { Gran Santiago, el } 20 \% \text { sufría acoso sexual en su trabajo, mientras que el } \\
84.3 \% \text { reconoce que ese comportamiento existe en el ámbito laboral. A su } \\
\text { vez, el } 80.5 \% \text { de las encuestadas señaló que el acoso sexual "es disimula- } \\
\text { do y cuesta mucho comprobarlo". }\end{array}$ \\
\hline Costa Rica $^{3}$ & 1997 & $\begin{array}{l}\text { Una encuesta sobre hostigamiento sexual, realizada a } 1489 \text { estudiantes } \\
\text { hombres y mujeres de } 50 \text { colegios de todas las regiones del país, reportó la } \\
\text { incidencia (al menos una vez) de distintos tipos de experiencias de acoso } \\
\text { sexual: cartas indeseadas de tipo sexual: } 13.6 \% \text {; llamadas molestas de tipo } \\
\text { sexual: } 33.5 \% \text {; comentarios ofensivos de tipo sexual, } 49.1 \% \text {; ofrecimiento de } \\
\text { mejorar notas a cambio de conductas no deseadas: } 6.4 \% \text {; que les hayan tocado } \\
\text { partes íntimas de su cuerpo sin su consentimiento: } 32.0 \% \text {; piropos, silbidos, } \\
\text { sonidos molestos: } 64.0 \% \text {; miradas sugestivas, mal intencionadas: } 58.6 \% \text {; } \\
\text { amenazas para aceptar conductas sexuales indeseadas: } 7.7 \% \text {. }\end{array}$ \\
\hline Costa Rica ${ }^{4}$ & 2005 & $\begin{array}{l}\text { Hasta el mes de julio de } 2005 \text {, la Defensoría de habitantes recibió } 40 \\
\text { denuncias por acoso sexual en el lugar de trabajo. }\end{array}$ \\
\hline El Salvador ${ }^{5}$ & $1998-2001$ & $\begin{array}{l}\text { Entre estos años, fueron denunciados } 1,354 \text { casos de acoso sexual, y en el } \\
93 \% \text { de éstos las víctimas fueron mujeres. }\end{array}$ \\
\hline El Salvador ${ }^{6}$ & 1999 & $\begin{array}{l}\text { En esta encuesta nacional, efectuada con una muestra de } 1,251 \text { hombres y } \\
\text { mujeres de zonas rurales y urbanas, y que abarcó todos los departamentos del } \\
\text { país, cuando se preguntó cuál era el problema que más afectaba a las mujeres } \\
\text { en el país, el acoso sexual y las violaciones ocuparon el tercer lugar. El } \\
21.7 \% \text { de la población femenina opinó que es el maltrato o la violencia } \\
\text { intrafamiliar; el } 9.4 \% \text { señaló la situación económica, un } 7.6 \% \text { dijo que era el } \\
\text { acoso sexual y las violaciones. }\end{array}$ \\
\hline
\end{tabular}




\begin{tabular}{|c|c|c|}
\hline El Salvador ${ }^{7}$ & 2002 & $\begin{array}{l}\text { En una encuesta con mujeres trabajadoras del sector maquila, el } 8 \% \\
\text { declaró haber sufrido algún tipo de acoso sexual, desde comentarios } \\
\text { sexuales a manoseo no deseados, lo cuales habitualmente fueron } \\
\text { realizados por los supervisores de las fábricas. }\end{array}$ \\
\hline Guatemala $^{8}$ & 2004 & $\begin{array}{l}\text { Una investigación con trabajadoras del sector maquila encontró que el } 5.4 \% \\
\text { manifestaba haber sido víctima de acoso sexual por parte de un compañero } \\
\text { de trabajo, y el } 5.1 \% \text { por parte de un jefe. }\end{array}$ \\
\hline Honduras $^{8}$ & 2004 & $\begin{array}{l}\text { Una investigación con trabajadoras del sector maquila encontró que el } 3.6 \% \\
\text { manifestaba haber sido víctima de acoso sexual por parte de un compañero } \\
\text { de trabajo, y el } 0.5 \% \text { por parte de un jefe. }\end{array}$ \\
\hline México $^{9}$ & 2003 & $\begin{array}{l}\text { El } 95 \% \text { de trabajadoras mexicanas reportaban haber sido víctimas de } \\
\text { acoso sexual. }\end{array}$ \\
\hline México $^{10}$ & 2003 & $\begin{array}{l}\text { Una investigación con } 160 \text { mujeres trabajadoras en cuatro sectores diferentes } \\
\text { encontró lo siguiente: } \\
-28 \% \text { no estaba familiarizado con el término "acoso sexual" } \\
\text { (consecuentemente, no podrían reconocerlo como un comportamiento dañino). } \\
-26 \% \text { reconoce que el acoso sexual es un comportamiento indeseado, pero no } \\
\text { saben nada más sobre éste. } \\
\text {-11\% piensa que sólo los actos sexuales agresivos o violentos constituyen acoso } \\
\text { sexual. } \\
\text { - } 53 \% \text { afirma no conocer ninguna ley que condene el acoso sexual. } \\
-47 \% \text { ha sufrido alguna forma de acoso sexual en el trabajo. De este porcentaje, } \\
\text { el } 25 \% \text { implicaba manoseo indeseado, el } 12 \% \text { recibió amenazas de sufrir } \\
\text { consecuencias si no cedía ante las demandas y el } 2 \% \text { sufrió asalto sexual. } \\
\text {-En el } 35 \% \text { de los casos los agresores fueron personas con posiciones superio- } \\
\text { res, mientras que en el } 23 \% \text {, fueron empleados del mismo nivel que la víctima; } \\
\text { en un } 20 \% \text {, los agresores fueron líderes gremiales o sindicales, en un } 15 \% \text { por } \\
\text { ciento fueron clientes y en un } 7 \% \text { fueron policías. }\end{array}$ \\
\hline Nicaragua $^{11}$ & 1995-1998 & $\begin{array}{l}\text { Según los registros policiales, en } 1995 \text { se registraron } 55 \text { casos de acoso } \\
\text { sexual; en } 1996,95 \text { casos; en } 1997,127 \text { casos; y hasta noviembre del } 1998 \text {, } \\
127 \text { casos. Por tanto, entre } 1995 \text { y } 1997 \text {, se duplicó el número de denuncias } \\
\text { por acoso sexual. }\end{array}$ \\
\hline Nicaragua $^{7}$ & 2002 & $\begin{array}{l}\text { En una encuesta con mujeres trabajadoras del sector maquila, el } 11.47 \% \\
\text { afirmó haber sufrido acoso sexual. A pesar de esto, se pudo comprobar que } \\
\text { encuentran muy difícil hablar sobre estos temas. Debido a eso, nueve de cada } \\
\text { diez casos no fueron denunciados. }\end{array}$ \\
\hline Nicaragua $^{12}$ & 2005 & $\begin{array}{l}\text { Entre Enero y Septiembre de } 2005 \text {, las Comisarías de la Mujer y la Niñez } \\
\text { atendieron } 2207 \text { delitos sexuales, de los cuales el } 11.1 \% \text { correspondían al delito } \\
\text { de acoso sexual. }\end{array}$ \\
\hline Panamáa ${ }^{13}$ & 1990 & $\begin{array}{l}\text { Una investigación con } 90 \text { mujeres funcionarias del sector público } \\
\text { encontró que aproximadamente un } 70 \% \text { de las encuestadas había sido } \\
\text { víctima de acoso sexual. }\end{array}$ \\
\hline Panamá ${ }^{14}$ & 1993 & $\begin{array}{l}\text { Una investigación con } 40 \text { mujeres, encontró que el } 41.03 \% \text { de mujeres } \\
\text { reconocían haber sido acosadas sexualmente. }\end{array}$ \\
\hline Panamáa ${ }^{15}$ & 1994 & $\begin{array}{l}\text { Esta investigación reveló que, de } 500 \text { trabajadoras del sector público y } \\
\text { privado que fueron entrevistadas, el } 53.6 \%(268) \text { había experimentado acoso } \\
\text { sexual. De estas } 268 \text {, solamente } 10 \text { lo denunciaron, y dichas denuncias } \\
\text { fueron inadvertidas en un } 50 \% \text { de los casos. }\end{array}$ \\
\hline Uruguay $^{16}$ & 1993 & $\begin{array}{l}\text { Un estudio con una muestra representativa de los hogares de Montevideo (396 } \\
\text { entrevistas) encontró que, entre las mujeres que trabajan, el } 4 \% \text { manifiesta } \\
\text { haberse sentido acosada sexualmente en el trabajo, mientras que un } 13 \% \\
\text { se ha sentido acosada en la calle. El } 7 \% \text { de las mujeres entrevistadas se ha } \\
\text { sentido hostigada sexualmente en otras situaciones. }\end{array}$ \\
\hline
\end{tabular}


En El Salvador, ha sido difícil encontrar estadísticas sobre acoso sexual, lo que reafirma la idea de que es una problemática a la que, por ignorancia o por conveniencia, no se le presta mucha atención. Sin embargo, la ONG feminista "Las Dignas" reporta que, según la Fiscalía General de la República, entre 1998 y 2001, fueron denunciados 1,354 casos de acoso sexual, y en el 93\% de éstos las víctimas fueron mujeres (Las Dignas, s/f).

En adición a todo esto, cabe mencionar que, si bien en la actualidad la mayoría de países cuenta con una legislación que sanciona el acoso sexual en los espacios laborales, su aplicación en países como El Salvador, todavía enfrenta graves deficiencias y la mayor parte de la población laboral desconoce su función, alcance e importancia (Bureau of Democracy, Human Rights, and Labor, 2006b; Las Dignas, 1999).

Es importante recalcar que la mayoría de estudios realizados sobre este tema, y de los cuales se obtienen las estadísticas sobre su prevalencia, son referentes al acoso sexual que se presenta en el ámbito laboral. Esto es entendible desde el punto de vista en que este ámbito ofrece mayores facilidades para realizar estudios de modo sistemático y, además, por la importancia que tiene el trabajo en la vida de las personas y la necesidad de procurar su desarrollo en una ambiente seguro y sin riesgos.

El trabajo es una actividad humana que debería tener como función primordial la obtención de lo necesario para vivir dignamente, y entre las condiciones inherentes a esto, además del ingreso económico, se contempla que el trabajo debe ser enriquecedor para la persona que lo realiza y permitirle el desarrollo de todo su potencial. Sin embargo, la realidad es muy diferente en El Salvador y otros países latinoamericanos: para las grandes mayorías el trabajo constituye una actividad para la subsistencia y, para otras tantas, el trabajo no sólo no es fuente de enriquecimiento sino que les somete a condiciones denigrantes que deben soportar por el miedo a perderlo y no poder encontrar otro. Esta situación es importante considerarla al tratar el acoso sexual, pues en muchos casos éste obliga a las víctimas a cambiar de trabajo, les impone la carga de soportarlo por el miedo a perder su empleo o, en ocasiones, incluso las ubica en riesgo de ser despedidas (Centro de Estudios de la Mujer, 1997; Instituto de la Mujer, 2006). Por ejemplo, en un estudio realizado en 2006, el Instituto de la Mujer de España reporta que, de las mujeres que habían sufrido acoso sexual en trabajos anteriores, el 51.9\% reconoce que esa situación ha influido en su cambio de trabajo. A su vez, una encuesta realizada en Chile por el Centro de Estudios de la Mujer (CEM) en 1993, reveló que el 32.2\% de una muestra de trabajadoras acosadas sexualmente renunció a su empleo después de ser objeto de tal conducta (CEM, 1997).

De igual manera, un estudio publicado por la Organización Internacional del Trabajo (OIT, 2002) acerca del trabajo infantil en El Salvador señala que, además de trabajar hasta 16 horas al día, a veces con sólo uno o dos días libres al mes, más del 60\% de las muchachas encuestadas denunciaron malos tratos físicos o psicológicos por parte de sus empleadores, y dentro de éstos el 10\% de las niñas trabajadoras domésticas reportó sufrir acoso sexual. Además, de las niñas que habían tenido trabajos anteriores en el ámbito doméstico, el 15.4\% señaló el acoso sexual como la causa para dejar el empleo anterior (Godoy, 2002).

Por otro lado, es menester recalcar que el acoso sexual también ocurre en áreas en las que se ha investigado mucho menos su presencia, como el plano educativo (OPS, 2003a; 
Witkowska, 2005), el religioso (Campos, 2004) o los cuerpos militares (Lipari y Lancaster, 2002; Quinn, 1996), entre otros. Dziech y Weiner, (citados por Charney y Russell, 1994) por ejemplo, analizaron varios estudios sobre la prevalencia del acoso sexual en ámbitos educativos realizados con diferentes metodologías, y concluyeron que entre el 20\% y el 30\% de estudiantes del sexo femenino son víctimas de acoso sexual mientras asisten a la universidad. Estas cifras son mayores para estudiantes graduadas/os que para no graduadas/os y, a su vez, las de éstas/os últimas son mayores que para las/os estudiantes de secundaria. Charney y Russell (1994) señalan que esto se puede deber a que para los/ as estudiantes graduados/as el contacto personal con sus supervisores es más cercano y porque dependen directamente de ellos para avanzar en sus estudios.

Tres estudios realizados en Estados Unidos a nivel nacional sobre el acoso sexual en la escuela, en los años 1992, 1993 y 2000, reflejan que la mayoría de las y los estudiantes (entre el 81\% y el 89\% de las chicas y entre el 60\% y el 79\% de los chicos) refieren haber sufrido alguna forma de acoso sexual durante su vida escolar (Witkowska, 2005). Y, a su vez, un estudio realizado por Komaromy y colaboradores (Charney y Russell, 1994) con 133 residentes en medicina interna, reveló que el 73\% de las mujeres y el 22\% de los hombres habían sido víctimas de acoso sexual, y que las mujeres eran más frecuentemente víctimas de sus formas severas. Aunque la muestra para esta investigación era pequeña, los resultados encontrados son apoyados por otras encuestas con estudiantes de medicina. Entre el 36 y el 52\% de estudiantes reportó acoso sexual durante sus estudios de medicina, con una prevalencia mucho mayor si el estudiante era del sexo femenino.

A partir de lo apuntado anteriormente, podemos señalar que, a pesar de las grandes diferencias que se reportan sobre la incidencia del problema en distintos países, el acoso sexual sigue siendo una problemática de interés para aquellas personas, disciplinas académicas e instituciones que están comprometidas a garantizar el bienestar de los seres humanos en todos los contextos y relaciones dentro de las cuales se desenvuelvan. Por tanto, para lograr erradicar un problema como éste, es de vital importancia construir una explicación profunda e integral, que sirva de base para diseñar mecanismos efectivos orientados a contrarrestarlo.

A continuación se presentan aquellos elementos que dan cuenta, desde la perspectiva psicosocial, de la manera en que se ha generado el acoso sexual y las construcciones sociales que le subyacen. Sea este último punto uno de los fines primordiales del presente trabajo, pues quitando el velo de "natural" a lo que ha sido histórica, social y políticamente construido -es decir, desideologizando la realidad- se puede dar un gran paso en el camino hacia la justicia. Y así, las víctimas podrán saber que no son culpables, que su situación de inequidad no es natural y, por tanto, que ésta puede ser transformada.

\section{Los cimientos del acoso sexual: violencia, ideología y discriminación por género}

El acoso sexual es una forma de violencia de género, la cual tiene sus raíces en la construcción social, cultural e histórica de la supuesta superioridad del hombre sobre la mujer. A partir de las definiciones de Koss y colaboradores, y de la conferencia Belém do Pará (Gaborit, 2005), se define aquí la violencia de género como toda acción de cualquier índole, experimentada por la mujer o la niña en razón de su sexo, cuyo propósito es perjudicarla, degradarla o 
despojarla de la capacidad para controlar su entorno, posibilitando su sometimiento físico y psicológico, tanto en el ámbito público como en el privado. La violencia contra las mujeres es usada tanto en un ambiente privado por un marido violento, como en conflictos bélicos a manera de instrumento de sometimiento -por ejemplo, la violación- (Gaborit, 2005). Otras manifestaciones de la violencia de género son la expulsión de adolescentes embarazadas o madres jóvenes de centros educativos, tráfico de mujeres, prostitución/embarazo forzado, privación de movilidad, inequidad salarial, y exclusión de cargos u oficios, por ejemplo, poca representación en los estratos más altos de organizaciones.

Para estudiar los fundamentos de la violencia de género desde la psicología social, hay que remitirse a lo que Martín-Baró (1985) llama "socialización sexual”, que es el proceso por el que la persona adquiere una identidad como hombre o mujer. Este proceso de socialización parte de características biológico-corporales entre ambos sexos, desde las que se construyen diferencias psíquico-sociales, en cuanto a roles, expectativas, aspiraciones y normas. Los niños y las niñas desarrollan un repertorio de conductas de dominación y sumisión, según sean de un sexo o de otro. De este modo, frente al sentido común, las diferencias entre sexos son inmodificables y legítimas, y por tanto hay una distribución de roles funcional y "natural". Pero la diversidad sexual da paso a la discriminación social, y la dimensión cultural del problema surge cuando se constata que las mujeres sufren, en mayor o menor medida, malos tratos a lo largo de su vida. Según datos de Las Dignas (2003), si bien gran parte de la violencia de género se da entre mujeres jóvenes, cualquier mujer, desde las recién nacidas hasta las ancianas, son o pueden llegar a ser víctimas. Las estadísticas muestran que desde pequeñas son maltratadas por ambos padres, sus hermanos y sus maestros; al crecer, por novios, compañeros de vida o esposos, y de adulta mayor, por parte de sus hijos, nietos y yernos (Las Dignas, 2003; FESAL citado por Gaborit, 2005).

La violencia de género está ligada al sexismo, o ideología de rol sexual, definido por Moya (citado por Expósito y Moya, 2005: 204) como las "actitudes acerca de los roles y responsabilidades considerados apropiados para hombres y mujeres, así como las creencias acerca de la relaciones que los miembros de ambas categorías deben mantener entre sí". En función de esta pertenencia, se asumen diferentes características, conductas, roles y actitudes, las cuales nutren de contenido el concepto descriptivo de "femenino" o "masculino" respectivamente. En la mayoría de los casos, sexismo y violencia de género se aplica a la conducta ejercida por los hombres contra las mujeres, en la que el género del agresor y el de la víctima van íntimamente unidos a la explicación de la discriminación y la violencia (Expósito y Moya, 2005). El sexismo cuenta con formas sutiles y encubiertas de expresión que pasan desapercibidas y se sumergen en explicaciones y justificaciones que buscan, en el fondo, perpetuar el tratamiento desigual hacia las mujeres. En esta línea, surge la teoría acerca de la ambivalencia hacia la mujer, que sugiere que las ideologías sexistas son un continuo, que abarca desde visiones paternalistas (sexismo benevolente) hasta el uso de la violencia (sexismo hostil). Estas dos formas se complementan y están muy difundidas en la cultura, reflejando y manteniendo la opresión de la mujer (DeSouza, Prior, Ribeiro, Mello y Camino, 2004).

Como marco general para dar lugar al sexismo, América Latina tiene un sistema patriarcal en el que se maneja el concepto de la mujer-objeto: la actitud y la creencia de que la mujer 
es poseíble, y que este carácter de objeto define la subjetividad femenina. En cuanto objeto puede moldearse, si fuese necesario, de forma violenta, para satisfacer metas o necesidades del otro. Y, tanto los guiones culturales como la socialización, engendran relaciones interpersonales de poder y control, que influyen en la internalización de valores, expectativas y comportamientos altamente genéricos. Por ejemplo, están las creencias de que la mujer se encuentra desprotegida y débil sin un hombre, y que ella posee, en menor grado, aquellos rasgos que caracterizan la esencia de lo humano: es menos racional, con menor capacidad de control del entorno; por tanto, es un ser humano inferior (Molpeceres, 2005).

Por otro lado, la definición de violencia desde la psicología social debe ser destacada, puesto que deja al descubierto elementos clave para la comprensión de las dinámicas de dominación y sumisión por género. La violencia es definida por Santacruz y Concha-Eastman (2001), como aquellas acciones intencionales que implican el uso de la fuerza o del poder, mediante la cual una o más personas producen daño físico, psicológico, sexual, en su libertad de movimiento, o la muerte a otra u otras personas, o a sí mismas, con un fin determinado. En un acto violento, hay dos características fundamentales (Savanije y Andrade, 2003): la intencionalidad, que se constituye por el motivo de la acción y su meta; y la justificación, que permite que el acto violento sea aceptado de acuerdo a las normas y patrones culturales del contexto en que se realiza (Martín-Baró, 1985).

Con respecto a la intencionalidad, la violencia en general, y la de género en particular, tiene un papel en la formación de códigos morales que rigen las relaciones entre la población y los sistemas de poder; suele ser valorada como un instrumento adecuado y necesario en ciertas situaciones, y los daños son metas aceptables. En El Salvador, la violencia forma parte importante del sistema de normas y valores sociales, formales e informales, que aceptan, toleran y retroalimentan las conductas violentas, y dictan pautas de relación entre las personas (Programa de las Naciones Unidas para el Desarrollo, 2003; Savanije y Andrade, 2003). Además de ser un instrumento que permite alcanzar un fin, es un carácter que impregna las relaciones interpersonales de los miembros de la sociedad.

El segundo elemento del acto violento, la justificación, viene dada por la ideología, un componente fundamental de la acción humana que refiere a ésta a una realidad basada en intereses sociales determinados (Martín-Baró, 1985). La persona posee esquemas cognoscitivos y valorativos, y ella los actúa, pero la explicación adecuada de sus acciones está en la sociedad de la que es miembro. La ideología cumple una serie de funciones: permite interpretar la realidad, suministra esquemas prácticos de acción, justifica y legitima el orden social existente como válido para todos (es decir, naturaliza lo que es histórico), y reproduce el sistema social establecido. De este modo, la violencia y la discriminación de género, acciones realizadas tanto por personas particulares como por colectivos e instituciones, tienen, ante todo, un componente ideológico esencial. Las diferencias naturales que existen entre hombres y mujeres han sido históricamente trasladadas al plano ideológico y así ha surgido la idea de que existe una superioridad masculina. Esta idea ha tenido y tiene aún en la actualidad, un gran respaldo cultural, y es apoyada y perpetuada por instituciones sociales. Es lo que Martín-Baró (1985: 373) define como contexto social posibilitador, "un marco de valores y normas, formales o informales, que acepte la violencia como una forma de comportamiento posible, e incluso la requiera". 
La justificación social de una distribución asimétrica del poder permite mantener ésta, porque produce una profecía autocumplida: los sujetos internalizan determinados preceptos y se comportan de acuerdo a ellos (Molpeceres, 2005). La ideología que responde a los intereses de los grupos dominantes configura las concepciones del sentido común de la gente, generando una eficaz dinámica de autoperpetuación. Primero, porque emplea el mecanismo de la "naturalización", la construcción histórica se vuelve algo "natural". Segundo, porque configura no sólo la mentalidad de los grupos a cuyos intereses sirve, sino a la sociedad en su conjunto. Se garantiza así que "los grupos dominados colaboren en la perpetuación de la estructura social que los oprime y los anula como sujetos de cambio; los oprimidos son instrumentalizados para el mantenimiento y la afirmación del dominio que sufren” (Molpeceres, 2005: 402).

La dinámica de violencia de género surge entonces como fruto de una visión trastocada del mundo, de una construcción cultural alterada que busca perpetuar los privilegios masculinos. La víctima acaba culpabilizada y el perpetrador como el que, inevitablemente, ejecuta lo que le es propio, por derecho o por rol asignado (Unger y Crawford citados por Gaborit, 2005).

La violencia de género "es probablemente la violación de los derechos humanos más frecuente y que afecta a un mayor número de personas (...) es, además, la violación de derechos humanos más oculta e impune, y de hecho no ha sido reconocida como tal hasta 1995" (Expósito y Moya, 2005: 201). Ni siquiera existe conciencia de que determinados hechos son violentos, y por tanto han llegado a reconocerse como algo normal e inevitable. Por la misma cultura de culpabilizar a la víctima, aún las mujeres que han sufrido malos tratos y lo reconocen como una violación a sus derechos, suelen tener miedo a denunciar y revelar ciertas situaciones.

De esta manera, se observa que el acoso sexual debe entenderse dentro del amplio espectro de conductas que constituyen la violencia de género. El daño provocado por un acto violento suele mesurarse en términos físicos (corporales y materiales) y, debido a que muchas veces el acoso sexual no llega a constituir una agresión que deja una huella física, no se considera como un comportamiento violento. Sin embargo, el daño también puede ser psicológico. E independientemente de la naturaleza de las consecuencias, es un daño de carácter simbólico, porque afecta negativamente a símbolos y representaciones determinadas (Expósito y Moya, 2005; Savanije y Andrade, 2003). En este caso, es la degradación de la figura de la mujer sólo por el hecho de ser mujer.

\section{Elementos para definir el acoso sexual}

El acoso sexual no ha comenzado a ser reconocido como un problema social sino hasta alrededor de 1974. En Estados Unidos, los primeros juicios por acusaciones de este tipo dieron paso a legislaciones sobre el acoso sexual en el trabajo, pues hasta entonces, conductas como los chantajes sexuales eran considerados "normales". Inicialmente, el término de acoso sexual fue un intento de los movimientos feministas por sintetizar las experiencias de un grupo determinado de mujeres, en un espacio y tiempo particulares. Este término implicaba la ejecución, por parte de los hombres, de conductas que superficialmente aparentaban ser 
sexuales, pero que de hecho constituían un ejercicio de poder. Con la aceptación de esta expresión, se reconocía el acoso como una agresión masculina en el trabajo, que asumía la forma de una conducta masculina no deseada y no correspondida, y que afirmaba el rol sexual de la mujer, por encima de su función como persona y como trabajadora (Wise y Stanley, 1994).

A lo largo de las últimas décadas, muchas definiciones sobre el acoso sexual han sido planteadas y aún en la actualidad no hay consenso sobre cuál de ellas es la que mejor representa el fenómeno en su totalidad. Generalmente, la definición tradicional de acoso sexual hace referencia al lugar de trabajo como espacio en el que ocurre. Por ejemplo, la Comisión de Igualdad de Oportunidades de Empleo de Estados Unidos (EEOC) conceptualiza el acoso sexual como una "forma de discriminación sexual que ocurre cuando una conducta física o verbal no deseada de naturaleza sexual afecta el trabajo individual, interfiere de manera irracional con el desempeño individual en el trabajo o crea un ambiente de trabajo intimidatorio, hostil u ofensivo" (Moyer y Nath citados por Expósito y Moya, 2005: 208).

En El Salvador, el artículo 165 del Código Penal salvadoreño, desde su modificación en el año 2003 (Bonilla, 2003), define y penaliza el acoso sexual de esta forma: "el que realice conducta sexual indeseada por quien la recibe, que implique frases, tocamiento, señas u otra conducta inequívoca de naturaleza sexual será sancionado con prisión de tres a cinco años”. En este país, únicamente el Ministerio de Medio Ambiente, a través de su Reglamento Interno (2001), consta de un instrumento legal contra el acoso sexual. El artículo 2 de dicho documento lo define como la realización de "conductas sexuales indeseadas por quien las recibe, que impliquen tocamientos u otras conductas inequívocas de naturaleza sexual, reiteradas o no y que provoque efectos perjudiciales en el desempeño laboral del denunciante o en su estado general de bienestar personal". Algunas manifestaciones de acoso sexual que especifica el documento, aunque no las únicas, son las siguientes: requerimiento de favores sexuales que impliquen promesa implícita o expresa de brindar un trato preferente respecto de la situación actual o futura de empleo o beneficios para quien recibe el acoso; amenaza implícita o expresa de causar castigos o daños físicos, psicológicos, sexuales o morales referidos a la situación actual o futura de empleo o beneficios de quien recibe el acoso; uso de palabras de naturaleza o contenido sexual, escritas, orales o gráficas, que resulten hostiles, humillantes u ofensivas para quien las recibe; acercamientos corporales u otras conductas físicas de naturaleza sexual, indeseadas y ofensivas para quien las reciba.

Otras definiciones son más abiertas e incluyen ámbitos distintos al laboral, como el educativo, el religioso, y el deportivo. Wise y Stanley (1994), desde el feminismo, han propuesto una definición concisa pero global: es una intrusión indeseada y no buscada por la mujer, en sus sentimientos, pensamientos, conductas, espacio, tiempo, energías y cuerpo, por parte de un hombre. Por otro lado, para Charney y Russell (1994), el acoso sexual es una forma de coerción sexual basada en el poder del acosador sobre algún aspecto de la vida de la víctima, y es una conducta que no necesariamente involucra fuerza física. Debe diferenciarse de otras formas de acoso que no involucran conductas de naturaleza sexual, y debe distinguirse también del flirteo, del galanteo, proposiciones de citas, y otros comportamientos que no sean percibidos con desagrado por la persona que los recibe. De hecho, como plantean estos autores, un factor clave en los casos de acoso sexual es que en ellos no están presentes los 
elementos de libertad de decisión y reciprocidad inherentes a las relaciones interpersonales normales.

Si bien se ha hecho avances significativos en el tema, el definir qué es acoso sexual aún plantea serios problemas. Uno de ellos es que, generalmente, la definición incluye aspectos subjetivos o perceptivos, como cuando se establece que son "conductas no deseadas por parte de quien las recibe". Además de esto, se requiere puntualizar aquellos factores que inciden en las percepciones y actitudes que se tienen respecto al acoso sexual: qué tan evidente es la conducta, con qué frecuencia ocurre, la relación del acosador con la víctima y las visiones propias de cada género, puesto que los hombres tienden a considerar que el atractivo sexual es el principal, y a veces único, motivo de acoso, mientras que las mujeres creen que es el poder (Wise y Stanley, 1994).

El problema de la percepción de qué es y qué no es acoso sexual se refleja en la investigación realizada por DeSouza et al. (2004), quienes estudiaron las reacciones al acoso sexual por parte de enfermeras brasileñas. Estos autores afirman que mucho de lo que los brasileños saben sobre acoso sexual viene de la prensa popular, la cual lo define como "el uso de la autoridad [poder] para obtener favores sexuales" ("Assédio", 1995, en DeSouza et al., 2004). En este sentido, las enfermeras que participaron en el estudio parecen percibir las formas menos severas de acoso sexual, como acoso de género y atención sexual no requerida (especificadas más adelante), como culturalmente "normales", debido a la rígida estructura patriarcal de los privilegios del hombre. A su vez, en este estudio se encontró que la mención de romance en una historia disminuía las respuestas punitivas de quienes fueron entrevistadas: el interés romántico del acosador hacia el blanco hizo que los avances sexuales parecieran apropiados y no coercitivos. Esto conlleva a que acosadores potenciales puedan enmascarar su comportamiento de asedio como cortejo o interés romántico, tratando de ser más privado e íntimo con el blanco. La literatura sobre acoso sexual apenas ha comenzado a explorar el nexo entre romance y acoso sexual. Como sostienen Glick et al. (citado por Páez et al., 2003), aún si las intenciones de los hombres están libres de hostilidad, puede manifestarse una forma de sexismo benevolente que deja a las mujeres, especialmente si los hombres involucrados ocupan un estatus social más alto, dominadas por medios placenteros, a cambio de que ellas reconozcan su lugar como objeto sexual.

Ante estas consideraciones, en algunas investigaciones se hace una diferenciación entre el acoso técnico y el acoso declarado. El primero se refiere al padecimiento de cualquiera de las situaciones definidas como acoso sexual, independientemente de que la víctima lo considere o no acoso sexual. Por el contrario, el segundo se entiende como las situaciones sufridas por una persona y que ella las considera como acoso sexual (Instituto de la Mujer, 2006).

Por otro lado, Expósito y Moya (2005) señalan que existen tres tipos de acoso sexual: a) Acoso de género: conductas relacionadas con el sexo, de tipo ofensivo, grosero, o despectivo, que sirven para expresar actitudes insultantes $\mathrm{u}$ ofensivas hacia las mujeres. Se dirigen hacia las mujeres por el hecho de ser mujeres; b) Atención sexual no deseada: atención y conductas sexuales que no son bien recibidas, ni han sido suscitadas y, por tanto, no son recíprocas; c) Coacción: intentos explícitos o implícitos de obtener cooperación sexual con 
la promesa de recompensas o la amenaza de castigos.

Sin embargo, aquí se sostiene que tanto el acoso de género como la atención sexual pueden ser dos componentes de un solo hecho. En muchas situaciones de acoso, al igual que en otras como la violación sexual, el presunto componente sexual es accesorio, y lo fundamental es reducir a otra persona al estado de objeto; el elemento buscado por el acosador es el poder (Wise y Stanley, 1994). De esta manera se puede afirmar que el acoso sexual es una forma de discriminación contra la mujer, como resultado de sistemas patriarcales que tratan a la mujer como objeto sexual (DeSouza et al., 2004). Precisamente uno de los modelos explicativos de esta problemática que mejor da cuenta de los diversos factores involucrados es el sociocultural. Para este modelo, el acoso sexual de las mujeres por los hombres es el resultado del estatus inferior de la mujer en el lugar de trabajo y en la sociedad en general. Por ejemplo, los trabajos de las mujeres han sido típicamente extensiones del rol de género femenino tradicional: "muchas mujeres pueden tener éxito en ciertas carreras, como enseñanza y enfermería, pero pueden ser excluidas al perseguir ocupaciones y roles dominados por el hombre" (DeSouza et al., 2004: 34).

De este modo, el acoso sexual es la herramienta que mantiene a las mujeres "en su lugar", de acuerdo a lo denominado como identidad de género, esto es, la identidad social que se deriva de la pertenencia a un grupo. Las personas se tratan entre sí en virtud de sus pertenencias grupales, y los miembros de grupos dominados o de bajo estatus suelen ser percibidos en función de su grupo de pertenencia y, por tanto, tienden a verse a sí mismos a la luz de sus características grupales en mayor medida que los miembros de grupos dominantes o de alto estatus (Lorenzi-Cioldi citado por Expósito y Moya, 2005). En este caso, los hombres, por el mero hecho de serlo, ya guardan relaciones de subordinador/subordinada con las mujeres, aun cuando éstas ocupen puestos jerárquicamente superiores. "Ser mujer implica tener como 'status primordial' el de 'mujer', independientemente de que sea neurocirujana, soldadora o ama de casa” (Wise y Stanley, 1994: 68).

Reiterar la condición de objeto de la mujer no es algo que ocurre exclusivamente en el ámbito laboral y privado. Cherish Kramarae (citado por Wise y Stanley, 1994) llevó a cabo un estudio sobre piropos hacia las mujeres en la vía pública, y propuso que los mismos se basan en la concepción masculina de que una mujer siempre está a la disposición de quien quiera, como propiedad pública. También concluyó que el halago sexual, los piropos y los silbidos en la calle, los toqueteos, las caricias y los abordajes verbales no tienen nada que ver con que un hombre sienta atracción sexual, sino con la expresión de poder y desprecio hacia la mujer. "La feminidad indica un perpetuo consentimiento de la mujer a las atenciones de cualquier hombre; a menos que un hombre determinado sea dueño de la propiedad en cuestión, se la considerará una propiedad pública” (Wise y Stanley, 1994: 191). Esta situación contrapone el derecho de la mujer a la intimidad contra el "derecho" de los hombres a piropearla y silbarle.

En San Salvador, ciudad capital de El Salvador, se encuentra vigente desde 1999 la Ordenanza Contravencional que, en teoría, sanciona económicamente diversos actos realizados en la vía pública, entre los que se cuenta el piropo. Con respecto a éste, la alcaldía municipal de San Salvador aplicaría sanciones de 300 a mil colones (actualmente, entre 30 y 100 
dólares estadounidenses) a la persona que dirija frases, gestos, ademanes, proposiciones indecorosas o realice tocamientos impúdicos en lugares públicos (Guevara, 1999).

Dos notas periodísticas referentes a esta ordenanza municipal mostraron la realidad (ver Gómez, 2004; Guevara, 1999). La primera se publicó en 1999, cuando aún no se había aprobado dicha ordenanza. Se realizó un sondeo entre mujeres, y todas las entrevistadas manifestaron que no existían medios adecuados para que la regulación pudiera ser llevada a la práctica. Por ejemplo, la ordenanza establece que las únicas autoridades que pueden imponer esquelas a los infractores son los agentes del Cuerpo de Agentes Metropolitanos (CAM); esto es, tiene que haber un policía municipal en el lugar de los hechos para sancionar. Y más allá de eso, como sostuvo otra entrevistada, "incluso los mismos policías municipales le dicen cosas obscenas a uno en las calles".

La segunda nota se publicó en el 2004, cuando la ordenanza ya llevaba alrededor de cuatro años en vigencia. A través de la crónica, escrita en primera persona por el periodista, se entreveía que la ordenanza distaba de cumplir su cometido: "un conductor de la ruta [de bus] 41-A, con su pecho velludo y gafas de cachada, hace relucir sus impulsos varoniles mientras hace un alto frente al semáforo. Hace sonar su bullicioso silbato cuando ve pasar a una elegante joven. Los delegados [de la policía] ni se percatan. Parecen más interesados en comprar un par de discos compactos clonados. (...) Al igual que el conductor avezado de la 41-A, [yo] lanzo un desacostrumbrado concierto de besos al aire al paso de unos elegantes y muy apretados 'blue jeans' y muy cerca de los indiferentes agentes del CAM (...). Una mujer que corría apresurada hacia su trabajo atrajo no sólo la mirada, sino que inspiró un desentonado pero insinuante canto de Arjona: 'Señora de las cuatro décadas...'. Pero esta vez el piropo no provenía de un transeúnte, sino de uno de los agentes [policiales] que cuida la entrada y salida del señor alcalde capitalino" (Gómez, 2004).

Las situaciones descritas rompen, hasta cierto punto, con la definición tradicional de acoso sexual, puesto que no se encuentran en el ámbito laboral y algunas de las acciones, como hacer sonar un silbato o cantar, no son explícitamente conductas de naturaleza sexual. La intención exacta de quien profiere el piropo y la percepción de éste de quien lo recibe sólo pueden suponerse, pero el argumento de la justificación no escapa, sobre todo si se tiene en cuenta el contexto patriarcal; la nota periodística de 1999 también contenía la opinión de un taxista: "yo no estaría de acuerdo en que se nos apliquen multas sólo a nosotros, ya que también hay algunas mujeres que se visten provocativamente y nos andan enseñando todo el cuerpo" (Guevara, 1999).

La "intención" se construye, socialmente, sobre la base de cómo se interpreta el significado de la conducta real, a partir de un significado atribuido y, en última instancia, se sanciona o avala el hecho según el marco de referencia o ideología. Las definiciones tradicionales de acoso sexual sostienen que, al final, es la mujer misma quien juzga si hubo o no acoso. Surge entonces la interrogante de hasta qué punto un hecho de esta naturaleza puede definirse en función de la percepción de quien lo comete y quién lo recibe. Como demostró el estudio de Souza et al. con las enfermeras brasileñas, el marco ideológico patriarcal se manifiesta tanto en hombres como en mujeres y, muchas veces, actos de acoso sexual serán calificado como "naturales" y esperables. De modo que deben tener más peso las creencias 
e interpretaciones del acosador y de la acosada, que las conductas reales.

De hecho, gran parte del acoso en la vía pública proviene de hombres, según Wise y Stanley (1994: 96), que creen tener el derecho automático de exigir a las mujeres su atención: "el acoso sexual no es en realidad sino la conducta perfectamente natural del hombre que se siente atraído hacia una mujer y procura relacionarse con ella. Por consiguiente, se argumenta, las mujeres deberíamos tomarlo como un halago, porque lo que se pone de manifiesto es simplemente el aprecio por nosotras y nuestros encantos". Estas autoras exponen casos en los que hombres piropean a mujeres (ellas mismas u otras) y las atacan verbalmente al no obtener las respuestas "simpáticas" que los piropeadores creen tener derecho a recibir.

Estas situaciones de acoso sexual en la vía pública también dejan en claro que, si bien en la mayoría de casos el acosador es del sexo masculino, es difícil saber las características exactas de quienes perpetran estos hechos. Como sostienen Expósito y Moya (2005: 212), el acoso sexual no es una aberración en el sentido de que "no es perpetrado por individuos desviados, ni constituye en la gran mayoría de los casos un problema psicológico” y, por lo tanto, los intentos por definir el perfil demográfico e interpersonal del típico acosador han fracasado. En el ámbito laboral en particular se ha reportado que suelen ser más frecuentemente compañeros de la víctima que personas en una posición de autoridad. No obstante, no todas las investigaciones concuerdan con este dato. Probablemente la situación sea más compleja debido a que pueden intervenir otros factores, como la severidad del acoso. En este sentido, algunos estudios reflejan que, a medida que aumenta la severidad de la conducta de acoso, aumenta considerablemente el protagonismo de las personas en niveles jerárquicos superiores como perpetradoras del acoso (Instituto de la Mujer, 2006). Aunque no se sabe mucho acerca de las características de las personas que acosan sexualmente, se ha logrado constatar en cierta medida que los acosadores suelen tener actitudes misóginas, hostilidad hacia las mujeres, visión de la sexualidad como lucha y conquista, y defienden la visión tradicional de los roles de género (Expósito y Moya, 2005).

\section{A manera de conclusión}

A partir de todo lo dicho anteriormente, surgen algunas observaciones que deben recalcarse. En primer lugar, se hace patente la necesidad de realizar investigaciones sobre la incidencia de este problema, que tengan criterios metodológicos similares, para poder comparar los niveles entre países, culturas o etnias diferentes. Este tipo de información puede abonar a la explicación de cómo los factores culturales e ideológicos del patriarcado y el machismo juegan un papel que propicia y ampara las situaciones de acoso. Asimismo, los gobiernos estatales deben procurar establecer entidades responsables de llevar un registro de las denuncias por este delito y de acompañar a las víctimas, tanto en el proceso judicial, como en el terapéutico. Esto se señala, ya que los gobiernos que cumplen con estas condiciones son la excepción más que la regla.

Por otra parte, puesto que este fenómeno se ha confinado conceptualmente como propio del lugar de trabajo, se ha explicado mayormente en términos de jerarquía, insistiendo que sólo incurren en él los hombres con poder institucional. Según Blumenthal (citado por DeSouza et $a l, 2004)$, el poder o estatus del acosador sobre quien sufre el acoso afecta la percepción 
del acoso sexual con el comportamiento iniciado por un superior, siendo percibido como más severo que el mismo comportamiento iniciado por un compañero de trabajo o alguien en similar posición.

Es imperativo reformular la definición de acoso sexual, de modo que ésta permita identificar objetivamente aquellas conductas de contenido sexual que atentan contra el espacio personal de una persona, por lo general del sexo femenino. Esta redefinición presenta algunas dificultades, porque debe superar las intrusiones extremas en ámbitos bien delimitados -como manoseos, en el ámbito laboral, por un superior- para incluir intromisiones más comunes y habituales -como los piropos, en la vía pública, por un desconocido- e ir más allá de caracterizar el problema superficialmente, como una acción meramente sexual (Wise y Stanley, 1994). Wise y Stanley (1994) proponen que, en la dinámica del acoso sexual, la "atracción sexual" es el fundamento y la "conducta sexual" el medio, pero la finalidad es el poder sobre la otra persona. En este sentido, Pryor y Strack (citado por Expósito y Moya, 2005) sostienen que existe conexión cognitiva entre poder y sexualidad, de modo que estar en una posición de poder sobre una mujer puede provocar excitación sexual. Y, en la cultura patriarcal, el hombre tiene, inherentemente, poder sobre la mujer, de modo que el acoso sexual puede considerarse una herramienta y un resultado de la dominancia masculina en la sociedad (DeSouza et al., 2004).

Algunos autores y autoras han planteado la interrogante de si el acoso sexual debe perfilarse como un acoso en el que se utiliza el sexo para lograr poder, o en el que se usa el poder para obtener sexo. Esto es, si este tipo de conductas debe entenderse como una conducta sexual o una conducta de poder. Pero la caracterización del acoso sexual no debe hacerse primordialmente en estos términos, poder o sexo, sino en términos más amplios: es una conducta violenta. Sea el daño físico, psicológico o simbólico, proviene de una ideología de rol sexual, construida históricamente, que justifica este daño, e incluso lo niega. Si uno de los efectos de la cultura patriarcal es la legitimación de las prerrogativas sexuales masculinas (ver tipologías machista y hembrista en Martín-Baró, 1985), entonces puede esperarse que los acosadores no serán necesariamente juzgados tan severamente (DeSouza et al, 2004).

Precisamente la ideología propia de la cultura patriarcal, a la vez que genera el acoso sexual, lo invisibiliza negando las víctimas. La mera discusión de este tema puede poner en riesgo la carrera de la mujer. De la cantidad de documentos que penalizan el acoso sexual, se desprende que hay una concepción que, para combatirlo, se debe recurrir a procedimientos formales, denuncias legales, etc. (Wise y Stanley, 1994), cuando en realidad, la vía legal es una solución con pocas probabilidades de éxito. El silencio de la víctima se mantiene por miedo al rechazo, a no ser creída, a resultar más ofendida, perjudicada y humillada (Expósito y Moya, 2005). Alexia Alvarado, abogada de CEMUJER (Campos, 2001), comenta que "[en El Salvador] el acoso sexual ahora es considerado un delito, pero ¿cuál es la realidad?, ¿qué pasa con esa mujer que denuncia a su jefe? Se encuentra con los problemas de procedimiento, donde la palabra de la mujer siempre está puesta en duda. Si la acusante pierde el caso, se frustra, arriesga su trabajo, se pone en evidencia y enfrenta a su familia". Y a pesar de que, como se dijo al principio, el lugar de trabajo ofrece ventajas para el estudio del acoso sexual, en América Latina es casi imposible reclutar mujeres en ocupaciones dominadas por el hombre, hay poco o ningún financiamiento para llevar a cabo 
investigaciones fuera de Estados Unidos (Barak, 1997), y el estudio de este tema no es bien visto en sociedades patriarcales (Souza et al, 2004).

Las ciencias sociales, y la psicología social en particular, tienen una deuda con quienes son partícipes, real o potencialmente, de esta problemática, en cuanto que ésta es un producto más de un sistema tradicionalista e intolerante hacia la diversidad y el cambio. Sobre esto hay dos puntos esenciales que deben tocarse: Primero, el estudio de este fenómeno desde y hacia otras poblaciones debe recibir igual atención, y aún está por verse hasta qué punto el acoso sexual hacia los hombres, y hacia personas de ambos sexos con orientación homosexual, puede plantearse en los mismos términos que el acoso sexual hacia las mujeres.

Segundo, hasta el momento el acercamiento a la contraparte del acoso sexual, a quien lo perpetra, se realiza desde la absolución o la culpabilización. La violencia de género se fundamenta en relaciones de poder, en las que el género masculino se perfila como el género hegemónico; poco se ha hecho para fomentar visiones alternativas a la masculinidad tradicional. Es necesario empezar por dejar de creer que la violencia masculina es inevitable. Movimientos como Men Can Stop Rape (mencanstoprape.org), en Estados Unidos, y Hombres Abolicionistas de la Prostitución (hombresabolicionistas.org), en España, buscan romper con el rol masculino tradicional. Mientras, por un lado, se sostenga que uno de los "valores" más altos que debe tener un "hombre de verdad" es el acumular experiencias sexuales, siendo la mujer solamente un "trofeo" para adquirir; y por otro se reproduzca la idea de que el valor primordial de la mujer es su cuerpo y su virginidad, y que si hay hombres que intentan "arrebatárselo" es porque no ha cuidado bien de él o porque lo ha exhibido demasiado (dando a entender que es "fácil" obtenerlo), poco podrá lograrse en la lucha contra el acoso sexual y otras formas de violencia sexual.

Algunos estudios (Leidig, 1992; Koss y cols., 1994; Rozee, 1993, en Gaborit, 2005) han demostrado que en las culturas que menos enfatizan la separación tradicional de roles masculinos y femeninos, hay menos violencia contra las mujeres que en aquellas donde los roles genéricos son relativamente rígidos. De modo que cuando las desigualdades de género se unen a la norma cultural que permite el uso de la violencia como medio de los poderosos para controlar a los débiles, y como medio de internalización de normas, se crea un ambiente que legitima la violencia hacia la mujer.

Finalmente, es responsabilidad de las ciencias sociales dejar en claro que el acoso sexual no debe considerarse simplemente como esperable ni normal; al contrario, está relacionado estrechamente con el estatus social inferior de la mujer, de modo que es un asunto de violencia y derechos humanos. En este sentido, solamente la construcción de una nueva masculinidad y una nueva feminidad, encaminada hacia el, hoy día, lejano horizonte de la equidad, es el camino para erradicar uno de los problemas más antiguos y graves en la historia de la humanidad: la discriminación de niñas, mujeres y adultas mayores.

\section{Notas}

1 Ver Unión del Personal Civil de la Nación (citado por Instituto Social y Político de la Mujer, s/f)

2 Ver Centro de Estudios de la Mujer (citado por Farías y Gómez, 1999). 
3 Instituto de Investigaciones Psicológicas de la Universidad de Costa Rica (citado por Radio Internacional Feminista - FIRE, s/f).

4 Ver Bureau of Democracy, Human Rights and Labor (2006a).

5 Ver Las Dignas (s/f)

6 Ver Instituto Universitario de Opinión Pública de la Universidad Centroamericana “José Simeón Cañas" (2000).

7 Ver Turner (2002)

8 Ver Arce (2006)

9 Ver OPS (2003)

10 Ver Trueba (2003)

11 Ver PNUD (1999a)

12 Ver Benítez (2006)

13 Ver Miller (citado por PNUD, 1999b)

14 Ver Miller (citado por PNUD, 1999b)

15 Ver Villareal (citado por PNUD, 1999b)

16 Ver Instituto Nacional de la Familia y la Mujer - UNICEF (citado por PNUD, 1999c)

\section{Referencias bibliográficas}

-ARCE, M. (2006). “Trabajadoras toleran abuso”, Noticias aliadas. 43, 7, 5-6.

-BENÍTEZ, G. (2006). Marco regulatorio e institucional para la prevención y la atención de la violencia doméstica y sexual y el VIH/ SIDA en Nicaragua [en línea]. Organización Panamericana de la Salud, Unidad Género, Etnia y Salud, [consultado el 28 de junio de 2006]. Disponible en Internet: www.paho.org/English/AD/GE/HIV-Nic-Profile-Jan06.pdf -BONILLA, A. (2003). “Delitos sexuales con mayores penas” El Diario de Hoy [en línea]. 26 de noviembre de 2003, [consultado el 25 de julio de 2006]. Disponible en Internet: http://www. elsalvador.com/noticias/2003/11/26/nacional/nacio11.html

-BUREAU OF DEMOCRACY, HUMAN RIGHTS, AND LABOR (2006a). Country Reports on Human Rights Practices - 2005. Costa Rica [en línea]. [Consultado el 26 de junio de 2006]. Disponible en Internet: http://www.state.gov/g/drl/rls/hrrpt/2005/61727.htm

-BUREAU OF DEMOCRACY, HUMAN RIGHTS AND LABOR (2006b). Country Reports on Human Rights Practices. El Salvador [en línea]. [Consultado el 17 de junio de 2006]. Disponible en Internet: http://www.state.gov/g/drl/rls/hrrpt/2005/61722.htm -CAMPOS, O. (2001). "Derechos de mujer", Revista Hablemos, El Diario de Hoy [en línea]. 2 de diciembre de 2001. [Consultado el 25 de julio de 2006]. Disponible en Internet: http:// www.elsalvador.com/hablemos/Ediciones/021201/actualidad.htm

-CAMPOS, O (2001). "Cuando la fe es manipulada”, Revista Hablemos, El Diario de Hoy [en línea]. 3 de octubre de 2001. [Consultado el 14 de julio de 2006]. Disponible en Internet: http://www.elsalvador.com/hablemos/2004/031004/031004-4.htm

-CENTRO DE ESTUDIOS DE LA MUJER (1997). El acoso sexual en el trabajo: Análisis de grupos focales [en línea]. Departamento de Estudios de la Dirección del Trabajo, Santiago de Chile. [Consultado el 18 de Julio de 2006]. Disponible en Internet: http://www.dt.gob.cl/ documentacion/1612/articles-59885_recurso_1_aporte_al_debate_2.pdf -CHARNEY, D. Y RUSSELL, R. (1994). "An overview of sexual harassment”. American Journal of Psychiatry, 151, 10-17.

-DESOUZA, E., PRIOR, J., RIBERIO, J., MELLO, J., y CAMINO, C. (2004). "Female Nurses' and Educators' Reactions to Sexual Harrasment Charges: A Cross-Cultural Perspective". Revista Interamericana de Psicología, 38, 33-40.

-EXPÓSITO, F. Y MOYA, M. (2005). "Violencia de género" en F. Expósito y M. Moya. (Coords.) Aplicando la Psicología Social. Madrid: Pirámide, 201-227. 
-FARÍAS, P. Y GÓMEZ, A. (1999). Acoso sexual en el trabajo. De la impunidad a la acción [en línea]. Santiago: Dirección del trabajo. [Consultado el 20 de julio de 2006]. Disponible en Internet: www.dt.gob.cl/documentacion/1612/articles-59901_recurso_7_aporte_al_debate_ 7.pdf

-GABORIT, M. (2005). “Los senderos del poder: violencia en contra de las mujeres", Estudios Centroamericanos (ECA), 681-682, 676-698, El Salvador.

-GEA 21. S.L., PERNAS, B., OLZA J., ROMÁN, M. Y LIGERO, J. (2000). El alcance del acoso sexual en el trabajo en España. Madrid: Paralelo.

-GODOY, O. (2002). El Salvador. Trabajo Infantil Doméstico: Una evaluación rápida [en línea]. [Consultado el 20 de julio de 2006]. Disponible en Internet: http:/www.ilo.org/public/ spanish/standards/ipec/simpoc/elsalvador/ra/domestic.pdf

-GÓMEZ, I. (2004). "Los tropiezos capitalinos", Revista Vértice, El Diario de Hoy [en línea]. 4 de julio de 2004. [Consultado el 29 de junio de 2006]. Disponible en Internet: http://www. elsalvador.com/vertice/2004/040704/cronica.html

-GONZÁLEZ, E. (1996). Acoso sexual. Buenos Aires: Desalma.

-GUEVARA, R. (1999). Sanciones contra la inmoralidad, El Diario de Hoy [en línea]. 23 de septiembre de 1999. [Consultado el 29 de junio de 2006]. Disponible en Internet: http://www. elsalvador.com/noticias/EDICIONESANTERIORES/septiembre23/VIDA

-INSTITUTO DE LA MUJER (2006). El acoso a las mujeres en el ámbito laboral [en línea]. [Consultado el 24 de julio de 2006]. Disponible en Internet: http:/www.mtas.es/mujer/ mujeres/estud_inves/acoso\%20sexual.pdf

-INSTITUTO SOCLAL Y POLÍTICO DE LA MUJER (s/f). Acoso sexual [en línea]. [Consultado el 25 de julio de 2006]. Disponible en Internet: http:/www.ispm.org.ar/acososexual.htm

-INSTITUTO UNIVERSITARIO DE OPINIÓN PÚBLICA DE LA UNIVERSIDAD CENTROAMERICANA "JOSÉ SIMEÓN CAÑAS" (2000). La situación de la mujer en El Salvador [en línea]. [Consultado el 13 de junio de 2006]. Disponible en Internet: http://www. uca.edu.sv/publica/iudop/2000/boletin2/bol200.htm

-THE IRISH PRESIDENCY OF THE EUROPEAN UNION - FGS CONSULTING Y MCGOLGAN, A., IPEU-FGS (2004). Report on sexual harassment in the workplace in EU member states [en línea]. [Consultado el 20 de julio de 2006]. Disponible en Internet: http://www. unece.org/stats/gender/publications/Multi-Country/SexualHarassmentReport.pdf

-LAS DIGNAS (s/f). Día Nacional contra la Violencia hacia las Mujeres. Mimeo.

-LAS DIGNAS (1999). ¿Yo sexista? Material de apoyo para una Educación No Sexista. San Salvador: Comunicación Publicitaria, $2^{\mathrm{a}}$ edición.

-LAS DIGNAS (2003). Sistematización sobre violencia sexual e intrafamiliar -años 1992 a 2000-. San Salvador: Equipo Maíz.

-LIPARI, R. y LANCASTER, A. (2003). Armed forces 2002: Sexual harassment survey [en línea]. [Consultado el 20 de julio de 2006]. Disponible en Internet: www.defenselink.mil/ news/Feb2004/d20040227shs1.pdf

-MARTÍN-BARÓ, I. (1985). Acción e Ideología. San Salvador: UCA Editores.

-MINISTERIO DE MEDIO AMBIENTE Y RECURSOS NATURALES, MARN (2001). Reglamento Interno Contra la Discriminación y el Acoso Sexual en el Ámbito Laboral del Ministerio de Medio Ambiente y Recursos Naturales [en línea]. [Consultado el 22 de julio de 2006]. Disponible en Internet: http://www.marn.gob.sv/genero.htm.

-MOLPECERES, M. A. (2005). "El pensamiento sobre la mujer en la obra de Ignacio MartínBaró”. En N. Portillo, M. Gabority J. Cruz (Compiladores). Psicología Social en la Posguerra: 
Teoría y Aplicaciones desde El Salvador. San Salvador: UCA Editores, 395-409.

-ORGANIZACIÓN PANAMERICANA DE LA SALUD, OPS (2003a). "La violencia sexual”. En Informe mundial sobre violencia y salud. Washington D.C.: OPS, 160-197.

-ORGANIZACIÓN PANAMERICANA DE LA SALUD, OPS (2003b). Violencia contra las mujeres [en línea]. [Consultado el 20 de julio de 2006]. Disponible en Internet: http://www. paho.org/Spanish/AD/GE/VAW2003sp.pdf

-PÁEZ D., ET AL. (2003). "Relaciones entre grupos, estereotipos y prejuicio”. En D. Páez, I. Fernández, S. Ubillos, y E. Zubieta. Psicología Social, Cultura y Educación. Madrid: Prentice Hall.

-PROGRAMA DE NACIONES UNIDAS PARA EL DESARROLLO, PNUD (1999a). Proyecto Regional RLA/97/014. Informes Nacionales sobre la situación de la Violencia de Género contra las Mujeres. Informe Nacional Nicaragua [en línea]. [Consultado el 19 de julio de 2006]. Disponible en Internet: www.bvs.org.ni/adolec/doc/nicaragua.pdf

-PROGRAMA DE NACIONES UNIDAS PARA EL DESARROLLO, PNUD (1999b). Proyecto Regional RLA/97/014. Informes Nacionales sobre la situación de la Violencia de Género contra las Mujeres. Informe Nacional Panamá [en línea]. [Consultado el 19 de julio de 2006]. Disponible en Internet: www.undp.org/rblac/gender/campaign-spanish/panamabigfile.pdf -PROGRAMA DE NACIONES UNIDAS PARA EL DESARROLLO, PNUD (1999c). Proyecto Regional RLA/97/014. Informes Nacionales sobre la situación de la Violencia de Género contra las Mujeres. Informe Nacional Uruguay [en línea]. [Consultado el 19 de julio de 2006]. Disponible en Internet: www.undp.org/rblac/gender/campaign-spanish/ugy.pdf -PROGRAMA DE LAS NACIONES UNIDAS PARA EL DESARROLLO, PNUD (2003). Informe sobre el desarrollo humano, El Salvador 2003. Desafíos y opciones en tiempos de globalización. El Salvador: PNUD.

-PROGRAMA DE LAS NACIONES UNIDAS PARA EL DESARROLLO, PNUD (2005). ¿̇Cuánto le cuesta la violencia a El Salvador? San Salvador: PNUD.

-QUINN, M. (1996). Sexual harassment in the australian defense force. Canberra: Department of Defense.

-RADIO INTERNACIONAL FEMINISTA - FIRE (s/f). Hostigamiento sexual en población adolescente escolarizada en Costa Rica [en línea]. [Consultado el 25 de julio de 2006]. Disponible en Internet: http://www.fire.or.cr/joven7.htm

-SANTACRUZ, M., CONCHA-EASTMAN, A. (2001). Barrio adentro. La solidaridad violenta de las pandillas. San Salvador: IUDOP.

-SAVANIJE, W. y ANDRADE EEKHOF, K. (2003). Conviviendo en la orilla. Violencia y exclusión social en el Área Metropolitana de San Salvador. San Salvador: FLACSO Programa El Salvador.

-TURNER, J. (2002). Seguridad e higiene en la industria de la maquila: Encuestas de El Salvador y Nicaragua [en línea]. [Consultado el 28 de julio de 2006]. Disponible en Internet: www.cawn.org/newsletter/15/sp_health_safety.html

-TRUEBA, L. (2003). A report on sexual harassment in the workplace in Mexico [en línea]. [Consultado el 19 de julio de 2006]. Disponible en Internet: www.laborrights.org/ projects/women/Mexico\%20report.pdf

-WISE, S., STANLEY, L. (1992). El acoso sexual en la vida cotidiana. Barcelona: Paidós. -WITKOWSKA, E. (2005). Sexual harassment in schools. Prevalence, structure and perceptions. Estocolmo: National Institute for Working Life. 